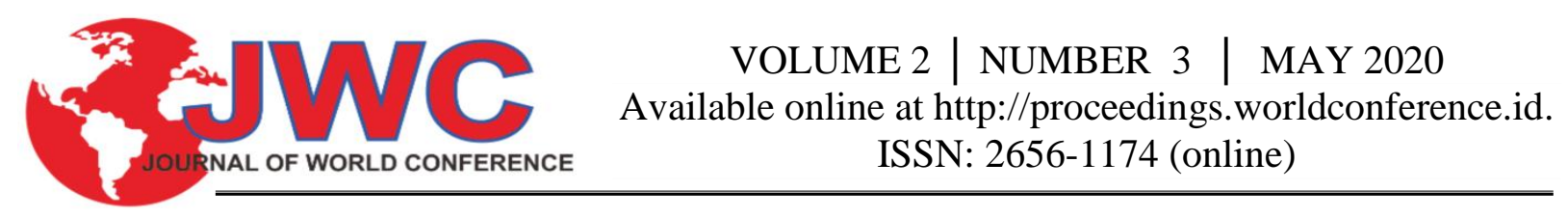

\title{
Drainage Capacity Analysis in The Area of Angke Jaya Tambora West Jakarta
}

\author{
Naufal Alfathan Bagas \\ Faculty of Engineering, University Mercu Buana, Bekasi, Indonesia, \\ bagasnaufalalf@gmail.co
}

\begin{abstract}
Drainage is used to reduce and remove excess water in an area so that the area can be optimally enabled. This research aims to analyze and examine the drainage problems in the Angke Jaya Tambora West Jakarta Housing area, the drain channels are poorly maintained and often flooding. The data used in this study are primary and secondary, in the planning of flood debt calculations used 2nd anniversary. For the calculation of flood discharge plan to use rational method and coupled with the discharge of flood household, and the result will be compared with existing conditions of drainage channels in the residential area Angke Jaya Tambora West Jakarta. Obtained flood discharge plan of $13.225 \mathrm{~m} 3 / \mathrm{sec}$, for the existing condition of drainage channels 5 channels enter the category is not safe because the condition of existing capacity of channels is less than flood discharge plan, namely channels A4, D5, D6, D8, and D9. The planning of the wells to accommodate the excess discharge flooding, and it takes 19 pieces of replacement wells on the A4 channel, 1 on the D5, D, D8, and D9 channels.
\end{abstract}

\section{Keywords}

Drainage, Hydrological Analysis, Flood Discharge Plans, Absorption Wells.

\section{Introduction}

Population growth in Indonesia is increasing every year and requires a lot of land to be used as dwellings, especially in urban areas. The narrowness of the land and the large number of residential developments and supporting facilities make many unnecessary land uses. The impact of a large number of human activities and lack of attention to the environment gives rise to many natural disasters, such as floods that most often occur in urban areas. During high rainfall it is not uncommon for water to flood in the streets and until flooding can occur, this is due to a lack of community attention to land use and drainage channels. Stagnant water or flooding that can also occur due to lack of functioning drainage channels or channels that are inadequate in accommodating water discharge. If no action is taken to overcome this problem it will disrupt community activities and hamper economic, socialandcultural development.

Planning and maintenance of drainage channels is needed to maintain and regulate the flow of water to create a healthy and comfortable environment. Drainage system in general can be interpreted as a prsarana that functions to drain excess water from an area to receiving water bodies such as rivers. Drainage can also be interpreted as a system of drainage of clean water and wastewater from residential areas, industries, agriculture, road bodies and other pavement surfaces, as well as channeling excess water in general, whether in the form of rainwater, wastewater or other dirty water that comes out of the area concerned to water bodies or artificial recharge buildings.

In this study, researchers will conduct research on the Angke Jaya Housing area, Angke Village, Tambora District, West Jakarta, which is a residential area that pays little attention to the drainage system and not infrequently the streets in the housing area are flooded during high rainfall.

\subsection{Identification Problems}

1. Full channel condition ( $10 \mathrm{~cm}$ height only)

2. The drainage on the channel is not smooth.

3. Sedimentation builds up in drainage channels.

4. There are a number of residential houses that have advanced to the street, covering the existing drainage, and making it difficult to clean the debris. 


\subsection{Problem Solving}

1. What is the condition of the drainage channels in the Angke Jaya Housing area, Angke Village, Tambora District, West Jakarta?

2. What is the flood discharge in the Angke Jaya Housing area, Angke Village, Tambora District, West Jakarta?

3. Do you need to plan for a new dimension of drainage in the Angke Jaya

Housing area, Kelurahan Angke, Tambora District, West Jakarta?

\subsection{Purpose and Objectives}

1. Knowing the condition of drainage channels in the area of Angke Jaya

Housing, Angke Village, Tambora District, West Jakarta.

2. Knowing the magnitude of flood discharge in the Angke Jaya Housing area, Angke Village, Tambora District, West Jakarta.

3. Knowing whether or not the new dimension of drainage channel planning is Needed in the Angke Jaya Housing area, Angke Village, Tambora District, West Jakarta.

\subsection{Benefits of Research}

At this writing the benefit gained is knowing whether the design of drainage channels in the Angke Jaya Housing area, Angke Village, Tambora District, West Jakarta is sufficient to accommodate flood plans that have been analyzed later, and concludes whether it is necessary to plan a new drainage channel in the Housing area Angke Jaya, Angke Village, Tambora District, West Jakarta.

\subsection{Scope and Limitation of Problems}

1. The location of the study was conducted in the area of Angke Jaya Housing, Angke Village, Tambora District, West Jakarta.

2. It only calculates the discharge of water from rainwater and household waste water.

3. Not calculating a budget plan.

4. Only calculates dimensions.

\subsection{Library Review}

Drainage which is derived from English drainage means to drain, drain, dispose, or drain water. In the field of civil engineering, drainage in general can be defined as a technical measure to reduce excess water, whether coming from rain water, seepage, or excess irrigation water from an area / land, so that the function of the area / land is not disrupted. Drainage can also be interpreted as an effort to control groundwater quality in relation to salinity (Suripin,2004:7) According to Bambang Triatmodjo (2015: 1), hydrology is the science related to water on earth, both regarding its occurrence, distribution and distribution, its properties and its relationship with its environment, especially with living things. Hydrological planning can be found in several activities such as planning and operating water buildings, water supply for various purposes (clean water, irrigation, fisheries, animal husbandry), hydroelectric power, flood control, erosion and sedimentation control, water transportation, drainage, controlling pollution, waste water, etc.

\section{Methodology}

\section{Study of literature}

At this stage what is done in this study is to identify problems in the drinase system that occur in the Angke Jaya Housing Area, Kel. Angke, Kec. Tambora, West Jakarta and literature study on the Drinase system as reference material and knowledge in the process of data collection, data processing, research results, to get conclusions in this study.

\section{Data collection}

The data needed in this study are primary data and secondary data, which will be explained as follows :

1) Primary Data 


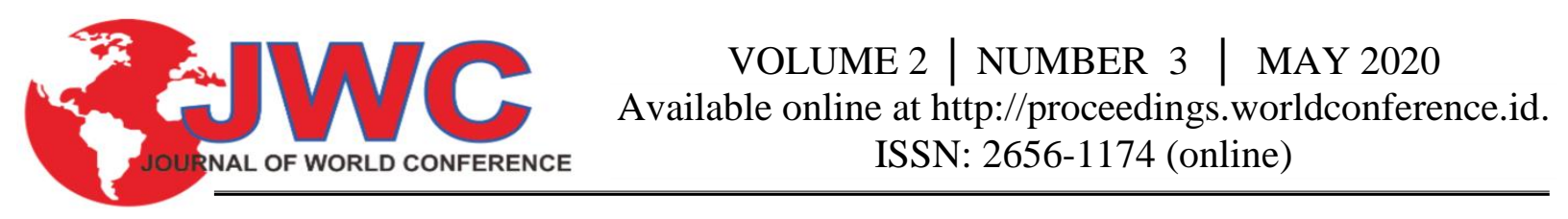

Primary data is population data to determine household wastewater discharge for the purpose of calculating flood discharge plans, and existing conditions of drainage channels including channel length, channel width, depth, channel elevation, channel type and channel catchment area to determine channel capacity requirements to accommodate incoming water discharge.

2) Second Data

Secondary data is data of minimum daily maximum rainfall for the last 10 years obtained from relevant agencies for the purposes of calculating flood discharge plans, and maps of the Angke Jaya Housing Area, Kel. Angke, Kec. Tambora, West Jakarta.

\section{Data Processing}

At this stage after all the necessary data has been collected, data processing will be carried out with the stages of the calculation of the flood discharge plan and the calculation of the capacity of the existing drainage channels.

\section{Data Processing Results}

After the results of data processing will be obtained the results of planned discharge and channel discharge, and if the channel discharge is greater than the planned flood discharge then the calculation of the dimensions of the new drainage channel is not carried out, and vice versa if the planned flood discharge is greater than the channel discharge it will be taken into account dimensions of new drainage channels.

\subsection{Research Methods}

The research methodology used in this thesis is an evaluative descriptive analysis, a method that evaluates objective conditions in a situation that is the object of research, and the object of the study is the drainage channel in the Angke Jaya Housing Area, Kel. Angke, Kec. Tambora, West Jakarta.

\subsection{Research Location and Time}

In this final project, the research will be conducted in August 2019 until September 2019. The research location is in the Angke Jaya Housing Area, Kel. Angke, Kec. Tambora, West Jakarta. The location map of the study can be seen in Picture 2.1.

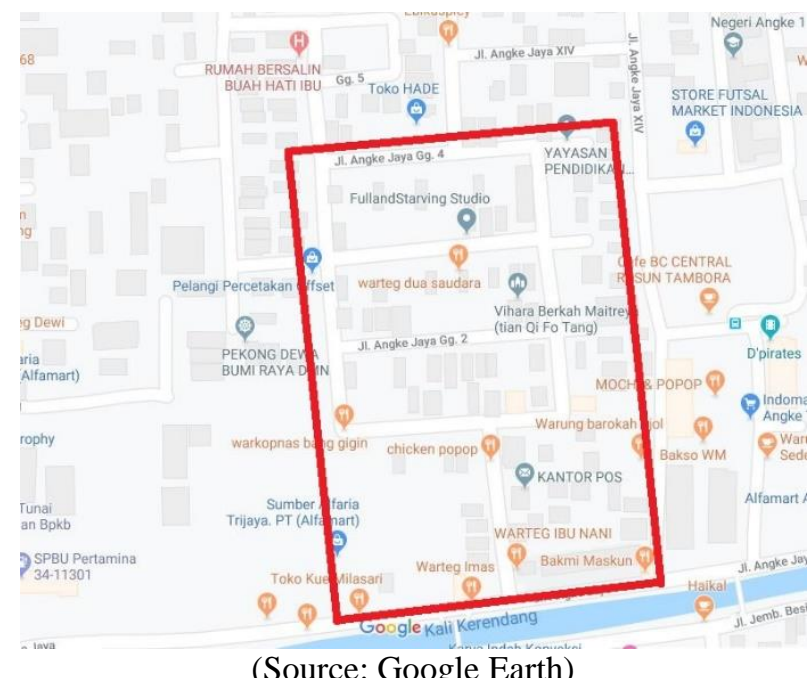

(Source: Google Earth)

Figure 2.1. Research Location Map

\section{Hydrological Analysis}

Hydrological analysis calculations are used to get flood discharge into the drainage channel being evaluated. The stages of the hydrological analysis calculation are as follows :

1. Calculate regional rainfall 
2. Analysis of the frequency of rainfall plans

3. Selection of distribution type

4. Test data compatibility

\subsection{Rainfall Area Analysis}

Rainfall data used is rainfall data for 10 years, namely from 2009 to 2018, rainfall data obtained from the Climatology and Geophysics Meteorological Agency.

Table 3.1. Daily / Month Maximum Rainfall Data ( $\mathrm{mm} /$ day)

\begin{tabular}{|c|c|c|c|c|c|c|c|c|c|c|c|c|}
\hline Tahum & Jan & Feb & Mar & Apr & Mei & Jun & Jul & Agt & Sept & Okt & Nov & Des \\
\hline 2009 & 148,9 & 87,1 & 35,4 & 26,9 & 73,4 & 22,4 & 15,7 & 6 & 15 & 16 & 39 & 48,5 \\
\hline 2010 & 82,4 & 88,3 & 52,9 & 13,7 & 85,2 & 44 & 28,5 & 18,6 & 56,1 & 52 & 29,8 & 69,3 \\
\hline 2011 & 31,1 & 58,1 & 30,6 & 6,5 & 62 & 58 & 51,3 & 10 & 2,8 & 34,3 & 78,5 & 66,4 \\
\hline 2012 & 48,9 & 60,9 & 60,3 & 50,1 & 43,6 & 21,9 & 25 & 0 & 24,6 & 0,1 & 75,1 & 59,4 \\
\hline 2013 & 117,8 & 53,7 & 52,7 & 34 & 47 & 46,4 & 37,7 & 71,8 & 0,8 & 41,8 & 37,2 & 81,5 \\
\hline 2014 & 154,1 & 284 & 165,3 & 10,9 & 165,3 & 43 & 33,7 & 71,8 & 53,7 & 0 & 46,4 & 34,1 \\
\hline 2015 & 133,4 & 247 & 54 & 30,7 & 25,1 & 25,2 & 2,5 & 0 & 16 & 0 & 0 & 87,4 \\
\hline 2016 & 34,3 & 108 & 38,8 & 112,7 & 16,4 & 75,5 & 43,4 & 42,3 & 39,5 & 49,3 & 29,1 & 6,6 \\
\hline 2017 & 82,9 & 148,6 & 26,5 & 41,5 & 44,3 & 53,4 & 19,8 & 1,9 & 43,2 & 23,3 & 39,1 & 48,1 \\
\hline 2018 & 66,3 & 100,5 & 129,6 & 69,3 & 14,7 & 12,2 & 0 & 46 & 15 & 54,1 & 39,8 & 11,9 \\
\hline
\end{tabular}

Source: Climatology and Geophysics Meteorological Agency

Table 3.2 Daily / Year Maximum Rainfall Data (mm / day)

References

\begin{tabular}{|c|c|}
\hline Tahun & $\begin{array}{c}\text { Curah Hujan } \\
\text { Maksimum }\end{array}$ \\
\hline 2009 & 148,9 \\
\hline 2010 & 88,3 \\
\hline 2011 & 78,5 \\
\hline 2012 & 75,1 \\
\hline 2013 & 117,8 \\
\hline 2014 & 284 \\
\hline 2015 & 247 \\
\hline 2016 & 112,7 \\
\hline 2017 & 148,6 \\
\hline 2018 & 129,6 \\
\hline
\end{tabular}

Source: Climatology and Geophysics Meteorological Agency

\subsection{Analysis of Frequency of Rainfall Plans}

Rainfall analysis of this plan is carried out to find out the maximum daily rainfall that will be used to calculate the flood discharge plan. The following calculations use the Normal Distribution, Normal Log Distribution, Log-Person III Distribution, and Gumbel Distribution.

\subsubsection{Normal Distribution}

The following calculation is the probability of rainfall for a 10 year return period with a Normal distribution.

Calculate the average value of variat $(\overline{\mathrm{X}})$

$$
\begin{aligned}
& \bar{X}=\frac{\sum X i}{n} \\
& \bar{X}=\frac{1430,5}{10}=143,05 \mathrm{~mm} / \text { hari }
\end{aligned}
$$


Table 3.3 Normal Distribution Calculation

\begin{tabular}{|r|r|r|r|r|r|r|r|}
\hline No. & Tahun & $\begin{array}{c}\text { Curah } \\
\text { Hujan } \\
\text { Kawasan } \\
(\mathbf{X i )}\end{array}$ & $\overline{\mathbf{X}}$ & $\mathbf{( X i}-\overline{\mathbf{X}})$ & $(\mathbf{X i}-\overline{\mathbf{X}})^{\mathbf{2}}$ & $(\mathbf{X i}-\overline{\mathbf{X}})^{\mathbf{3}}$ & $(\mathbf{X i}-\overline{\mathbf{X}})^{\mathbf{4}}$ \\
\hline 1 & 2009 & 148,9 & 143,05 & 5,85 & 34,22 & 200,20 & 1171,18 \\
\hline 2 & 2010 & 88,3 & 143,05 & $-54,75$ & 2997,56 & $-164116,55$ & 8985380,94 \\
\hline 3 & 2011 & 78,5 & 143,05 & $-64,55$ & 4166,70 & $-268960,65$ & 17361409,72 \\
\hline 4 & 2012 & 75,1 & 143,05 & $-67,95$ & 4617,20 & $-313738,91$ & 21318558,93 \\
\hline 5 & 2013 & 117,8 & 143,05 & $-25,25$ & 637,56 & $-16098,45$ & 406485,94 \\
\hline 6 & 2014 & 284 & 143,05 & 140,95 & 19866,90 & 2800239,91 & 394693814,94 \\
\hline 7 & 2015 & 247 & 143,05 & 103,95 & 10805,60 & 1123242,38 & 116761045,39 \\
\hline 8 & 2016 & 112,7 & 143,05 & $-30,35$ & 921,12 & $-27956,07$ & 848466,66 \\
\hline 9 & 2017 & 148,6 & 143,05 & 5,55 & 30,80 & 170,95 & 948,79 \\
\hline 10 & 2018 & 129,6 & 143,05 & $-13,45$ & 180,90 & $-2433,14$ & 32725,71 \\
\hline \multicolumn{2}{|c|}{$\mathbf{2}$} & $\mathbf{1 4 3 0 , 5 0}$ & & $\mathbf{0 , 0 0}$ & $\mathbf{4 4 2 5 8 , 5 9}$ & $\mathbf{3 1 3 0 5 4 9 , 6 8}$ & $\mathbf{5 6 0 4 1 0 0 0 8 , 2 1}$ \\
\hline
\end{tabular}

Source: Calculation Analysis Results

Frequency factor (KT) values for the 10 year return period are obtained from the Gauss Reduction Variable Value table, so the calculation of the probability of rainfall returns for the 10 year return period with the Normal distribution is as follows :

$$
\begin{aligned}
& X_{T}=\bar{X}+K_{T} \cdot S \\
& X_{10}=143,05+(1,28) \cdot(70,126) \\
& X_{10}=232,81 \mathrm{~mm} / \text { day }
\end{aligned}
$$

Calculation of rainfall with a return period with the Normal distribution can be seen in table 4.4

Table 3.4 Calculation of Rainfall for Birthday of T Year with Normal Distribution

\begin{tabular}{|c|c|c|c|c|}
\hline Periode Ulang T (tahun) & $\overline{\mathbf{X}}$ & $\mathbf{S}$ & $\mathbf{K}_{\mathbf{T}}$ & $\mathbf{X}_{\mathbf{T}}$ \\
\hline 2 & 143,05 & 70,126 & 0 & 143,05 \\
\hline 5 & 143,05 & 70,126 & 0,84 & 201,9558 \\
\hline 10 & 143,05 & 70,126 & 1,28 & 232,8113 \\
\hline 20 & 143,05 & 70,126 & 1,64 & 258,0566 \\
\hline 50 & 143,05 & 70,126 & 2,05 & 286,8083 \\
\hline 100 & 143,05 & 70,126 & 2,33 & 306,4436 \\
\hline
\end{tabular}

Source: Calculation Analysis Results

\subsubsection{Log Normal Distribution}

Following is the calculation of the rainfall probability re-distribution period of the Normal Log. Calculate the average value of variat $(\log \mathrm{X})$

$\log \overline{\mathrm{X}}=\frac{\sum \log \mathrm{X}}{\mathrm{n}}$

\begin{tabular}{|c|c|c|c|c|c|c|c|c|}
\hline No & Tahun & $\mathbf{x}$ & $\underset{\mathbf{X}}{\log }$ & $\underset{\bar{X}}{\log }$ & $\begin{array}{c}\log \\
X- \\
\log \\
\bar{X}\end{array}$ & $\begin{array}{l}(\log X- \\
\log \bar{X})^{2}\end{array}$ & $\begin{array}{l}(\log X- \\
\log \bar{X})^{3}\end{array}$ & $\left(\begin{array}{c}(\log X-\log \\
\bar{X})^{4}\end{array}\right.$ \\
\hline 1 & 2009 & 148,90 & 2,17 & 2,16 & 0,02 & 0,0003030 & 0,00000527 & 0,000000092 \\
\hline 2 & 2010 & 88,30 & 1,95 & 2,16 & $-0,21$ & 0,0439016 & $-0,00919858$ & 0,001927353 \\
\hline 3 & 2011 & 78,50 & 1,89 & 2,16 & $-0,26$ & 0,0679218 & $-0,01770167$ & 0,004613378 \\
\hline 4 & 2012 & 75,10 & 1,88 & 2,16 & $-0,28$ & 0,0783149 & $-0,02191625$ & 0,006133217 \\
\hline 5 & 2013 & 117,80 & 2,07 & 2,16 & $-0,08$ & 0,0071137 & $-0,00059999$ & 0,000050604 \\
\hline 6 & 2014 & 284,00 & 2,45 & 2,16 & 0,30 & 0,0887030 & 0,02641845 & 0,007868221 \\
\hline 7 & 2015 & 247,00 & 2,39 & 2,16 & 0,24 & 0,0562682 & 0,01334732 & 0,003166105 \\
\hline 8 & 2016 & 112,70 & 2,05 & 2,16 & $-0,10$ & 0,0107255 & $-0,00111077$ & 0,000115036 \\
\hline 9 & 2017 & 148,60 & 2,17 & 2,16 & 0,02 & 0,0002733 & 0,00000452 & 0,000000075 \\
\hline \multirow[t]{2}{*}{10} & 2018 & 129,60 & 2,11 & 2,16 & $-0,04$ & 0,0018389 & $-0,00007886$ & 0,000003382 \\
\hline & $\Sigma$ & 1430,50 & 21,14 & 21,55 & $-0,41$ & 0,36 & $-0,01$ & 0,023877463 \\
\hline
\end{tabular}

$\log \bar{X}=\frac{21,14}{10}=2,114 \mathrm{~mm} /$ day

Table 3.5 Calculation of Normal Log Distribution

Source: Calculation Analysis Results 
Table 3.6 Calculation of Rainfall for Birthday of T Year with Normal Log Distribution

\begin{tabular}{|c|c|c|c|c|}
\hline Periode Ulang T (tahun) & $\log \bar{X}$ & $\mathbf{S}$ & $\mathbf{K}_{\mathbf{T}}$ & $\mathbf{Y}_{\mathbf{T}}$ \\
\hline 2 & 2,114 & 0,199 & 0 & 2,114 \\
\hline 5 & 2,114 & 0,199 & 0,84 & 2,28116 \\
\hline 10 & 2,114 & 0,199 & 1,28 & 2,36872 \\
\hline 20 & 2,114 & 0,199 & 1,64 & 2,44036 \\
\hline 50 & 2,114 & 0,199 & 2,05 & 2,52195 \\
\hline 100 & 2,114 & 0,199 & 2,33 & 2,57767 \\
\hline
\end{tabular}

Source: Calculation Analysis Results

\subsubsection{Log Person III Distribution}

Following is the calculation of the probability of rainfall with the Log Pearson III distribution.

Table 3.7 Calculation Log Pearson III Distribution

\begin{tabular}{|c|c|c|c|c|c|c|c|c|}
\hline No & Tahun & \multicolumn{1}{|c|}{$\mathbf{X}$} & $\begin{array}{c}\mathbf{L o g} \\
\mathbf{X}\end{array}$ & $\begin{array}{c}\mathbf{L o g} \\
\overline{\boldsymbol{X}}\end{array}$ & $\begin{array}{c}\mathbf{L o g} \mathbf{X} \\
-\mathbf{L o g}\end{array}$ & $\begin{array}{c}\mathbf{L} \mathbf{L} \mathbf{X}-\mathbf{L o g} \\
\overline{\mathbf{X}})^{2}\end{array}$ & $\begin{array}{c}\mathbf{L o g} \mathbf{X}- \\
\mathbf{L o g} \overline{\mathbf{X}})^{\mathbf{3}}\end{array}$ & $\begin{array}{c}(\mathbf{L o g} \mathbf{X}- \\
\mathbf{L o g} \overline{\mathbf{X}})^{4}\end{array}$ \\
\hline 1 & 2009 & 148,90 & 2,17 & 2,16 & 0,02 & 0,0003030 & 0,00000527 & 0,000000092 \\
\hline 2 & 2010 & 88,30 & 1,95 & 2,16 & $-0,21$ & 0,0439016 & $-0,00919858$ & 0,001927353 \\
\hline 3 & 2011 & 78,50 & 1,89 & 2,16 & $-0,26$ & 0,0679218 & $-0,01770167$ & 0,004613378 \\
\hline 4 & 2012 & 75,10 & 1,88 & 2,16 & $-0,28$ & 0,0783149 & $-0,02191625$ & 0,006133217 \\
\hline 5 & 2013 & 117,80 & 2,07 & 2,16 & $-0,08$ & 0,0071137 & $-0,00059999$ & 0,000050604 \\
\hline 6 & 2014 & 284,00 & 2,45 & 2,16 & 0,30 & 0,0887030 & 0,02641845 & 0,007868221 \\
\hline 7 & 2015 & 247,00 & 2,39 & 2,16 & 0,24 & 0,0562682 & 0,01334732 & 0,003166105 \\
\hline 8 & 2016 & 112,70 & 2,05 & 2,16 & $-0,10$ & 0,0107255 & $-0,00111077$ & 0,000115036 \\
\hline 9 & 2017 & 148,60 & 2,17 & 2,16 & 0,02 & 0,0002733 & 0,00000452 & 0,000000075 \\
\hline 10 & 2018 & 129,60 & 2,11 & 2,16 & $-0,04$ & 0,0018389 & $-0,00007886$ & 0,000003382 \\
\hline & $\mathbf{\Sigma}$ & $\mathbf{1 4 3 0 , 5 0}$ & $\mathbf{2 1 , 1 4}$ & & $-\mathbf{0 , 4 1}$ & $\mathbf{0 , 3 6}$ & $-\mathbf{0 , 0 1}$ & $\mathbf{0 , 0 2 3 8 7 7 4 6 3}$ \\
\hline
\end{tabular}

Source: Calculation Analysis Results

The steps in using the Log Person III distribution are as follows :

Calculate the average price $(\log X)$

$\log \overline{\mathrm{X}}=\frac{\sum \log \mathrm{X}}{\mathrm{n}}$

$\log \bar{X}=\frac{21,14}{10}=2,114 \mathrm{~mm} /$ day

Calculating Standard Deviation Price (S)

$\mathrm{S}=\left[\frac{\sum_{\mathrm{n}=1}^{\mathrm{n}}\left(\log \mathrm{X}_{\mathrm{i}}-\log \overline{\mathrm{X}}\right)^{2}}{\mathrm{n}-1}\right]^{0,5}$

$=\left[\frac{0,36^{2}}{9}\right]^{0,5}$

$=0,199$

Calculate the coefficient of skewness

$\mathrm{G}=\frac{\sum_{\mathrm{n}=1}^{\mathrm{n}}\left(\log X_{\mathrm{i}}-\overline{\log X}\right)^{3}}{(\mathrm{n}-1)(\mathrm{n}-2) \mathrm{s}^{3}}$

$G=\frac{10 \times-0,01^{3}}{9 \times 8 \times(0,199)^{3}}=-0,0000176$

Calculates the rain or flood logarithm with a return period $\mathrm{T}$

The $\mathrm{K}$ value (Interpolation) is a standardized variable for $\mathrm{X}$, the magnitude of which depends on the coefficient of G, can be seen in the table K Value for the Distribution of Person Log III. 
Table $3.8 \mathrm{~K}$ value (interpolation)

\begin{tabular}{|c|c|c|c|}
\hline \multirow{3}{*}{$\begin{array}{c}\text { Periode } \\
\text { Ulang }\end{array}$} & \multicolumn{3}{|c|}{ G } \\
\hline & $-0,1$ & $\begin{array}{c}- \\
0.0000176\end{array}$ & 0,0 \\
\hline & \multicolumn{3}{|c|}{$\mathbf{K}$} \\
\hline 2 & 0,0165 & 0,00825 & 0,000 \\
\hline 5 & 0,846 & 0,844 & 0,842 \\
\hline 10 & 1,27 & 1,276 & 1,282 \\
\hline 25 & 1,7155 & 1,73325 & 1,751 \\
\hline 50 & 1,998 & 2,0245 & 2,051 \\
\hline
\end{tabular}

Source: Calculation Analysis Results

$$
\begin{aligned}
& \log X_{T}=\log \bar{X}+\text { K.s } \\
& \log X_{T}=2,114+0,00825 \times 0,199 \\
& \log X_{T}=2,116 \\
& X_{T}=130,617 \mathrm{~mm} / \text { day }
\end{aligned}
$$

Table 3.9 XT Calculation

\begin{tabular}{|c|c|c|c|c|}
\hline $\begin{array}{c}\text { Periode } \\
\text { Ulang }\end{array}$ & Kemencengan & $\mathbf{K}$ & $\log \mathbf{X}_{\mathbf{T}}$ & $\mathbf{X}_{\mathbf{T}}$ \\
\hline 2 & & 0,00825 & 2.116 & 130,617 \\
5 & & 0,844 & 2,281956 & 191,406 \\
10 & $-0,0000176$ & 1,276 & 2,367924 & 233,305 \\
25 & & 1,73325 & 2,45891675 & 287,685 \\
50 & & 2,0245 & 2,5168755 & 328,757 \\
\hline
\end{tabular}

Source: Calculation Analysis Results

\subsubsection{Gumbel Distribution}

\begin{tabular}{|c|c|c|c|c|c|c|c|}
\hline No. & Tahun & $\begin{array}{c}\text { Curah } \\
\text { Hujan } \\
\text { Kawasan } \\
(\mathbf{X i}) \\
\end{array}$ & $\overline{\mathrm{x}}$ & $(\mathbf{X i}-\overline{\mathbf{X}})$ & $(\mathbf{X i}-\overline{\mathbf{X}})^{2}$ & $(\mathbf{X} \mathbf{i}-\overline{\mathbf{X}})^{3}$ & $(\mathbf{X i}-\overline{\mathbf{X}})^{4}$ \\
\hline 1 & 2009 & 148,90 & 143,05 & 5,85 & 34,22 & 200,20 & 1171,18 \\
\hline 2 & 2010 & 88,30 & 143,05 & $-54,75$ & 2997,56 & $-164116,55$ & 8985380,94 \\
\hline 3 & 2011 & 78,50 & 143,05 & $-64,55$ & 4166,70 & $-268960,65$ & 17361409,72 \\
\hline 4 & 2012 & 75,10 & 143,05 & $-67,95$ & 4617,20 & $-313738,91$ & 21318558,93 \\
\hline 5 & 2013 & 117,80 & 143,05 & $-25,25$ & 637,56 & $-16098,45$ & 406485,94 \\
\hline 6 & 2014 & 284,00 & 143,05 & 140,95 & 19866,90 & 2800239,91 & 394693814,94 \\
\hline 7 & 2015 & 247,00 & 143,05 & 103,95 & 10805,60 & 1123242,38 & 116761045,39 \\
\hline 8 & 2016 & 112,70 & 143,05 & $-30,35$ & 921,12 & $-27956,07$ & 848466,66 \\
\hline 9 & 2017 & 148,60 & 143,05 & 5,55 & 30,80 & 170,95 & 948,79 \\
\hline 10 & 2018 & 129,60 & 143,05 & $-13,45$ & 180,90 & $-2433,14$ & 32725,71 \\
\hline \multicolumn{2}{|r|}{$\Sigma$} & 1430,50 & & 0,00 & 44258,59 & 3130549,68 & 560410008,21 \\
\hline
\end{tabular}

Calculate the average value of variat $(\log X)$

$$
\begin{aligned}
& \bar{X}=\frac{\sum \mathrm{Xi}}{\mathrm{n}} \\
& \bar{X}=\frac{1430,5}{10}=143,05 \mathrm{~mm} / \text { hari }
\end{aligned}
$$

Table 3.10. Gumbel Distribution Calculation

Source: Calculation Analysis Results 
Yn, Sn, and Ytr values are obtained from the Reduce Mean (Yn), Reduced Standard Deviation (Sn), and Reduced Variate (YTr) tables. Calculation of the probability value of rainfall for a 10 year return period with the Gumbel distribution is as follows :

$$
\begin{aligned}
X_{\operatorname{Tr}} & =\bar{X}+\frac{Y_{\operatorname{Tr}}-Y_{n}}{S_{n}} S \\
X_{10} & =143,05+\frac{2,2510-0,4952}{0,9496} \times 70,126 \\
& =272,712 \mathrm{~mm} / \text { day }
\end{aligned}
$$

\subsubsection{Selection of Distribution Type}

Statistical parameters in the selection of this type of distribution needed are Standard Deviation (S), Skewness Coefficient (Cs), Kurtosis Measurement (Ck) and Variation Coefficient (Cv).

\begin{tabular}{|c|c|c|}
\hline Jenis Distribusi & Syarat & Hasil \\
\hline Normal & $\begin{array}{l}\mathrm{C}_{\mathrm{s}} \approx 0 \\
\mathrm{C}_{\mathrm{k}} \approx 3\end{array}$ & $\begin{array}{l}C_{5}=-0,206 \\
C_{k}=0,341\end{array}$ \\
\hline Log Normal & $\begin{array}{l}\mathrm{Cs}_{\mathrm{s}}=\mathrm{Cv}^{3}+3 \mathrm{C}_{\mathrm{v}} \\
\mathrm{C}_{\mathrm{k}}=\mathrm{Cv}^{8}+6 \mathrm{Cv}^{6}+15 \mathrm{Cv}^{4}+16 \mathrm{Cv}^{2}+3\end{array}$ & $\begin{array}{l}C_{5}=-0,575 \\
C_{k}=0,335\end{array}$ \\
\hline Gumbel & $\begin{array}{l}\mathrm{C}_{\mathrm{s}}=1,14 \\
\mathrm{C}_{k}=5,40\end{array}$ & $\begin{array}{l}C_{s}=-0,206 \\
C_{k}=0,341\end{array}$ \\
\hline Log Person III & $\begin{array}{l}\mathrm{C}_{\mathrm{s}} \neq 0 \\
\text { Atau selain nilai diatas }\end{array}$ & $\begin{array}{l}C_{5}=-0,575 \\
C_{k}=0,335\end{array}$ \\
\hline
\end{tabular}
Here are the results of the calculation :

Table 3.11. Distribution Type Selection Parameters

Source: Calculation Analysis Results

From the calculation results presented in table 4.11, it can be concluded that the type of distribution that can be taken is the Log Person III distribution.

\subsection{Data Match Test}

\subsubsection{Chi Square Test}

Before carrying out the calculation of the data suitability test, first carry out data processing, which is to sort data from the largest to the smallest as presented in table 4.12.

Table 3.12. Daily / Year Maximum Rainfall Data (mm / day)

\begin{tabular}{|c|c|}
\hline Tahun & $\begin{array}{c}\text { Curah Hujan } \\
\text { Maksimum }\end{array}$ \\
\hline 2009 & 148,9 \\
\hline 2010 & 88,3 \\
\hline 2011 & 78,5 \\
\hline 2012 & 75,1 \\
\hline 2013 & 117,8 \\
\hline 2014 & 284 \\
\hline 2015 & 247 \\
\hline 2016 & 112,7 \\
\hline 2017 & 148,6 \\
\hline 2018 & 129,6 \\
\hline
\end{tabular}

Source: Calculation Analysis Results

The next stage is to calculate the distribution class $(\mathrm{G})$, which is as follows :

$\mathrm{G}=1+3,33 \log (\mathrm{n})$

$\mathrm{G}=1+3,33 \log (10)$ 
$\mathrm{G}=1+3.33(1)=4.33 \approx 5$

The distribution class used 10 pieces of data is 5 . As an interval class that is used every 20\%. Data intervals are taken from the reset period as follows :

The results of the calculation of the distribution class above are then included as class interval classes in each probability distribution.

From the calculation of the distribution of Log Person III are as follows:

$\mathrm{S}=0.199$

$\mathrm{G}=-0.0000176$

From table 2.2 the interpolation is done so that the $\mathrm{K}$ value for each interval is as follows:

$\mathrm{K} 5=0.842001$

$\mathrm{K} 2,5=0.140336$

$\mathrm{K} 1,67=-0,37048$

$\mathrm{K} 1.25=-0.842$

Following is the calculation of the class interval for the distribution of Log Person III

$\log$ X5 $=+$ K5. S.

$=2.114+(0.842001 .0 .199)$

$=2,282$

$\mathrm{X} 5=191,426$

$\log X 2,5=+$ K5. S

$=2.114+(0.140336 .0 .199)$

$=2,142$

$\mathrm{X} 2,5=138.68$

$\log \mathrm{X} 1,67=+\mathrm{K} 5 . \mathrm{S}$.

$=2.114+(-0.37048$. 0.199)

$=2,041$

$\mathrm{X} 1,67=109,901$

$\log \mathrm{X} 1.25=+\mathrm{K} 5$. S.

$=2,114+(-0,842.0,199)$

$=1,947$

$\mathrm{X} 1.25=88,512$

Table 3.13. Chi Square Test

\begin{tabular}{|c|c|c|c|c|c|}
\hline \multirow{2}{*}{ No } & \multirow{2}{*}{ Sub Kelas } & \multicolumn{2}{|c|}{ Jumlah Data } & \multirow{2}{*}{ Oi-Ei } & \multirow{2}{*}{$\boldsymbol{\chi}^{\mathbf{2}}=\frac{\left(\boldsymbol{O}_{i}-\boldsymbol{E}_{\boldsymbol{i}}\right)^{\mathbf{2}}}{\boldsymbol{E}_{\boldsymbol{i}}}$} \\
\cline { 3 - 4 } & $\mathrm{Oi}$ & $\mathbf{E i}$ & & 0.5 \\
2 & $\mathrm{P}<88,512$ & 3 & 2 & 1 & 2 \\
2 & $88,512<\mathrm{P}<109,901$ & 0 & 2 & -2 & 0.5 \\
3 & $109,901<\mathrm{P}<138,68$ & 3 & 2 & 1 & 0 \\
4 & $138,68<\mathrm{P}<191,426$ & 2 & 2 & 0 & 0 \\
5 & $\mathrm{P}>191,426$ & 2 & 2 & 0 & 3 \\
\hline & Jumlah & 10 & 10 & & \\
\hline
\end{tabular}

Source: Calculation Analysis Results

$E i=\frac{n}{k}=\frac{10}{5}=2$

$(D K)=5-(2+1)=2$

From Table 4.13 it is known, based on Table 2.7 the critical value for the Chi-squared distribution at the degree of confidence $(\alpha)=0.05$ or $5 \%$ obtained value Because the Pearson III log distribution equation can be accepted.

\subsubsection{Smirnov Kolmogorov Test}

The results of calculations can be seen in table 4.14, an example of calculation using data in 2008, which is as follows: 
a. Sorting rain data from large to small can be seen in table 4.12, and look for opportunities with the opportunity formula as follows :

$$
\begin{aligned}
& \mathrm{P}(\log \mathrm{X})=\frac{\mathrm{m}}{(\mathrm{n}+1)} \\
& \mathrm{P}(\log \mathrm{X})=\frac{1}{(10+1)}=0,091
\end{aligned}
$$

b. Look for the value of $\mathrm{P}(\mathrm{X}<)$ :

$$
\begin{aligned}
\mathrm{P}(\log \mathrm{X}<) & =1-\mathrm{P}(\log \mathrm{X}) \\
& =1-0,091=0,909
\end{aligned}
$$

c. Look for the value $\mathrm{f}(\mathrm{t})$ :

$$
f(t)=\frac{\log X-\log \bar{X}}{S}=\frac{2,113-2,114}{0,199}=-0,007
$$

d. the value of $\mathrm{P}^{\prime}(\log X)$ is searched using the area table under the normal curve of $\mathrm{f}(\mathrm{t})$ with the value :

\begin{tabular}{|c|c|c|c|c|c|c|c|c|c|}
\hline Tahun & M & $\mathrm{x}$ & $\begin{array}{c}\text { Log } \\
\mathrm{X}\end{array}$ & $\mathrm{P}(\log \mathrm{X})$ & $\mathrm{P}^{\prime}(\log \mathrm{X}<)$ & $\begin{array}{r}f(t)= \\
(\log X \\
-\log X / s\end{array}$ & $P^{\prime}(\log X)$ & $\mathrm{P}^{\prime}(\log \mathrm{X}<)$ & D \\
\hline \multirow[t]{11}{*}{1} & 2 & 3 & 4 & 5 & $6=1-$ Kolom 5 & 7 & 8 & $\begin{array}{c}9=1-\text { Kolom } \\
8\end{array}$ & $10=6-9$ \\
\hline & 1 & 148,90 & 2,173 & 0,091 & 0,909 & 0,296 & 0,111 & 0,889 & 0,020 \\
\hline & 2 & 88,30 & 1,946 & 0,182 & 0,818 & $-0,844$ & 0,222 & 0,778 & 0,040 \\
\hline & 3 & 78,50 & 1,895 & 0,273 & 0,727 & $-1,101$ & 0,333 & 0,667 & 0,060 \\
\hline & 4 & 75,10 & 1,876 & 0,364 & 0,636 & $-1,198$ & 0,444 & 0,556 & 0,080 \\
\hline & 5 & 117,80 & 2,071 & 0,455 & 0,545 & $-0,215$ & 0,556 & 0,444 & 0,101 \\
\hline & 6 & 284,00 & 2,453 & 0,545 & 0,455 & 1,705 & 0,667 & 0,333 & 0,122 \\
\hline & 7 & 247,00 & 2,393 & 0,636 & 0,364 & 1,400 & 0,778 & 0,222 & 0,142 \\
\hline & 8 & 112,70 & 2,052 & 0,727 & 0,273 & $-0,312$ & 0,889 & 0,111 & 0,162 \\
\hline & 9 & 148,60 & 2,172 & 0,818 & 0,182 & 0,292 & 1,000 & 0,000 & 0,182 \\
\hline & 10 & 129,60 & 2,113 & 0,909 & 0,091 & $-0,007$ & 1,111 & $-0,111$ & 0,202 \\
\hline
\end{tabular}

$$
\begin{aligned}
& \mathrm{P}(\log \mathrm{X})=\frac{\frac{\mathrm{m}}{(\mathrm{n}-1)}}{\mathrm{P}(\log \mathrm{X})}=\frac{1}{(10-1)}=0,111 \\
& \mathrm{Up} \text { to value }{ }^{\prime}(\log X): \\
& \mathrm{P}^{\prime}(\log \mathrm{X}<) \\
& =1-\mathrm{P}^{\prime}(\log \mathrm{X}) \\
& =1-0,111=0,889
\end{aligned}
$$

Find the D value using the formula:

$$
\mathrm{D}=\mathrm{P}(\log \mathrm{X}<)-\mathrm{P}^{\prime}(\log \mathrm{X}<)=0,909-0,889=0,020
$$

Table 3.14. Calculation Results of the Smirnov Kolmogorov Test Distribution Person Log III

Source: Calculation Analysis Results

From Table 4.14. above it can be concluded that Dmax $=0.202$ on the 9th sequence data. By using Table 2.8 . Critical value of D0 for Smirnov-Kolmogorov test with a degree of confidence of $5 \%$ and $n=10$, then obtained $\mathrm{D} 0=0.41$. Because the value of Dmax $<\mathrm{D} 0$ then Pearson log distribution distribution III can be accepted.

\subsection{Calculation of Flood Discharge Plan}

The design of flood discharge calculation is carried out to get the flood discharge entering the drainage.

\subsubsection{Flow Coefficient}

Flow coefficient $(\mathrm{C})$ is a constant price, a ratio between the rain that flows on the surface and the rain water that falls. In the Angke Jaya Tambora Housing Area of West Jakarta, the study was included in the character of multiunit housing (combined), so that the value obtained in accordance with table 2.9 was 0.60 to 0.75 and the highest value was taken, 0.75 . 


\subsubsection{Calculation of Time of Concentration of Channels}

The results of the calculation of the complete concentration time are presented in table 4.16

Tabel 3.15. Channel Concentration Time (tc)

\begin{tabular}{|c|c|c|c|c|c|c|c|c|c|c|c|}
\hline \multirow{2}{*}{\begin{tabular}{c|} 
No. \\
Saluran
\end{tabular}} & $\mathrm{L}$ & \multirow{2}{*}{ S } & \multirow{2}{*}{$\mathrm{n}$} & \multirow{2}{*}{\begin{tabular}{|c|} 
to \\
menit \\
\end{tabular}} & \multirow{2}{*}{\begin{tabular}{|c|} 
Asal \\
$\mathrm{m} 2$ \\
\end{tabular}} & \multirow{2}{*}{\begin{tabular}{|c|}
$\mathrm{A}$ \\
$\mathrm{m} 2$ \\
\end{tabular}} & \multirow{2}{*}{$\begin{array}{l}\mathrm{P} \\
\mathrm{m} \\
\end{array}$} & \multirow{2}{*}{$\begin{array}{l}\mathrm{R} \\
\mathrm{m} \\
\end{array}$} & \multirow{2}{*}{$\begin{array}{c}\mathrm{V} \\
\mathrm{m} / \mathrm{dt}\end{array}$} & \multirow{2}{*}{\begin{tabular}{|c|} 
td \\
menit \\
\end{tabular}} & \multirow{2}{*}{$\begin{array}{c}\text { tc } \\
\text { menit }\end{array}$} \\
\hline & $\mathrm{m}$ & & & & & & & & & & \\
\hline A1 & 102,50 & 0,02 & 0,01 & 22,46 & 0,60 & 0,60 & 2,60 & 0,23 & 3,75 & 0,46 & 22,92 \\
\hline A2 & 69,33 & \begin{tabular}{|l|} 
\\
\end{tabular} & 0,01 & 17,67 & 0,25 & 0,25 & 1,40 & 0,18 & 2,68 & 0,43 & 18,10 \\
\hline A3 & 125,70 & \begin{tabular}{|l|}
0,01 \\
\end{tabular} & 0,01 & 43,14 & \begin{tabular}{|l|}
0,48 \\
\end{tabular} & 0,48 & 2,00 & 0,24 & 2,46 & 0,85 & 43,99 \\
\hline A4 & 240,05 & 0,00 & 0,01 & 113,86 & 0,40 & 0,40 & 1,80 & 0,22 & 1,69 & 2,37 & 116,22 \\
\hline B1 & 125,70 & \begin{tabular}{|l|}
0,01 \\
\end{tabular} & 0,01 & 43,14 & 0,48 & 0,48 & 2,00 & 0,24 & 2,46 & 0,85 & 43,99 \\
\hline B2 & \begin{tabular}{|c|}
69,33 \\
\end{tabular} & \begin{tabular}{|l|}
0,01 \\
\end{tabular} & 0,01 & 17,67 & 0,25 & 0,25 & 1,40 & 0,18 & 2,68 & 0,43 & 18,10 \\
\hline B3 & \begin{tabular}{|l|}
128,50 \\
\end{tabular} & \begin{tabular}{|l|}
0,01 \\
\end{tabular} & 0,01 & 44,59 & \begin{tabular}{|l|}
0,48 \\
\end{tabular} & 0,48 & 2,00 & 0,24 & 2,43 & 0,88 & 45,47 \\
\hline B4 & 52,60 & 0,02 & 0,01 & 11,68 & 0,40 & 0,40 & 1,80 & 0,22 & 3,61 & 0,24 & 11,92 \\
\hline C1 & 128,50 & 0,01 & 0,01 & 44,59 & 0,48 & 0,48 & 2,00 & 0,24 & 2,43 & 0,88 & 45,47 \\
\hline $\mathrm{C} 2$ & 69,33 & \begin{tabular}{|l|}
0,01 \\
\end{tabular} & 0,01 & 17,67 & 0,30 & 0,30 & 1,70 & 0,18 & 2,70 & 0,43 & 18,10 \\
\hline C3 & 125,00 & \begin{tabular}{|l|}
0,01 \\
\end{tabular} & 0,01 & 42,78 & 0,48 & 0,48 & 2,00 & 0,24 & 2,47 & 0,84 & 43,63 \\
\hline $\mathrm{C} 4$ & 52,60 & 0,02 & 0,01 & 11,68 & \begin{tabular}{|l|}
0,40 \\
\end{tabular} & 0,40 & 1,80 & 0,22 & 3,61 & 0,24 & 11,92 \\
\hline D1 & 306,13 & 0,00 & 0,01 & \begin{tabular}{|l|l|}
163,97 \\
\end{tabular} & \begin{tabular}{|l|}
0,56 \\
\end{tabular} & 0,56 & 2,30 & 0,24 & 1,59 & 3,21 & 167,17 \\
\hline D2 & 306,13 & 0,00 & 0,01 & 163,97 & \begin{tabular}{|l|}
0,36 \\
\end{tabular} & 0,36 & 1,80 & 0,20 & 1,40 & 3,65 & 167,62 \\
\hline D3 & 31,20 & 0,03 & 0,01 & 5,34 & 0,20 & 0,20 & 1,40 & 0,14 & 3,49 & 0,15 & 5,48 \\
\hline D4 & 52,60 & 0,02 & 0,01 & 11,68 & 0,48 & 0,48 & 2,00 & 0,24 & 3,80 & 0,23 & 11,91 \\
\hline D5 & 31,00 & 0,03 & 0,01 & 5,28 & 0,16 & 0,16 & 1,20 & 0,13 & 3,35 & 0,15 & 5,44 \\
\hline D6 & 31,00 & 0,03 & 0,01 & 5,28 & \begin{tabular}{|l|}
0,16 \\
\end{tabular} & 0,16 & 1,20 & 0,13 & 3,35 & 0,15 & 5,44 \\
\hline D7 & 52,60 & \begin{tabular}{|l|l|}
0,02 \\
\end{tabular} & 0,01 & 11,68 & 0,48 & 0,48 & 2,00 & 0,24 & 3,80 & 0,23 & 11,91 \\
\hline D8 & 25,00 & 0,04 & 0,01 & 3,83 & 0,12 & 0,12 & 1,10 & 0,11 & 3,26 & 0,13 & 3,95 \\
\hline D9 & 25,00 & 0,04 & 0,01 & 3,83 & 0,12 & 0,12 & 1,10 & 0,11 & 3,26 & 0,13 & 3,95 \\
\hline D10 & 52,60 & 0,02 & 0,01 & 11,68 & 0,48 & 0,48 & 2,00 & 0,24 & 3,80 & 0,23 & 11,91 \\
\hline D11 & 51,25 & 0,02 & 0,01 & 11,23 & \begin{tabular}{|l|}
0,56 \\
\end{tabular} & 0,56 & 2,20 & 0,25 & 4,01 & 0,21 & 11,44 \\
\hline D12 & 51,25 & 0,02 & 0,01 & 11,23 & \begin{tabular}{|l|}
0,56 \\
\end{tabular} & 0,56 & 2,20 & 0,25 & 4,01 & 0,21 & 11,44 \\
\hline D13 & 208,00 & 0,00 & 0,01 & 91,83 & \begin{tabular}{|l|}
0,36 \\
\end{tabular} & 0,36 & 1,80 & 0,20 & 1,69 & 2,05 & 93,88 \\
\hline D14 & $\mid 101,50$ & \begin{tabular}{|l|}
0,01 \\
\end{tabular} & 0,01 & 31,30 & \begin{tabular}{|l|}
0,56 \\
\end{tabular} & 0,56 & 2,30 & $\begin{array}{ll}0,24 \\
\end{array}$ & 2,76 & 0,61 & 31,92 \\
\hline D15 & 101,50 & 0,01 & 0,01 & 31,30 & \begin{tabular}{|l|}
0,56 \\
\end{tabular} & 0,56 & 2,30 & 0,24 & 2,76 & 0,61 & 31,92 \\
\hline
\end{tabular}

Source: Calculation Analysis Results

\subsubsection{Calculation of Intensity of Rainfall Area}

The return period that will be used in the calculation of rainfall intensity is a return period of 2 years.

Rainfall intensity is calculated using the mononobe formula with the concentration time value (tc).

Calculation of rainfall intensity on channel A1 is as follows:

$\mathrm{XT}=130,617 \mathrm{~mm}$ day

$\mathrm{TC}=22.92$ minutes

$$
\begin{aligned}
& I=\left(\frac{X_{2}}{24}\right) \cdot\left(\frac{24}{t c}\right)^{\frac{2}{3}} \\
& I=\left(\frac{130,617}{24}\right) \cdot\left(\frac{24}{22,92 / 60}\right)^{\frac{2}{3}}=86,011 \mathrm{~mm} / \text { hour }
\end{aligned}
$$

The results of a complete rainfall intensity calculation are presented in table 4.16. 
Table 3.16. Rainfall Intensity (I)

\begin{tabular}{|c|c|c|c|c|}
\hline No & $\mathrm{X}_{\mathrm{T}}$ & $\mathrm{t}_{\mathrm{c}}$ & $\mathrm{t}_{\mathrm{c}}$ & $\mathrm{I}$ \\
\hline Saluran & $\mathrm{mm} /$ hari & menit & jam & $\mathrm{mm} /$ jam \\
\hline A1 & 130,617 & 22,920 & 0,382 & 86,011 \\
\hline A2 & 130,617 & 18,100 & 0,302 & 100,673 \\
\hline A3 & 130,617 & 43,990 & 0,733 & 55,692 \\
\hline A4 & 130,617 & 116,220 & 1,937 & 29,141 \\
\hline B1 & 130,617 & 43,990 & 0,733 & 55,692 \\
\hline B2 & 130,617 & 18,100 & 0,302 & 100,673 \\
\hline B3 & 130,617 & 45,470 & 0,758 & 54,477 \\
\hline B4 & 130,617 & 11,920 & 0,199 & 132,998 \\
\hline C1 & 130,617 & 45,470 & 0,758 & 54,477 \\
\hline C2 & 130,617 & 18,100 & 0,302 & 100,673 \\
\hline C3 & 130,617 & 43,630 & 0,727 & 55,998 \\
\hline C4 & 130,617 & 11,920 & 0,199 & 132,998 \\
\hline D1 & 130,617 & 167,170 & 2,786 & 22,870 \\
\hline D2 & 130,617 & 167,620 & 2,794 & 22,829 \\
\hline D3 & 130,617 & 5,480 & 0,091 & 223,277 \\
\hline D4 & 130,617 & 11,910 & 0,199 & 133,073 \\
\hline D5 & 130,617 & 5,440 & 0,091 & 224,370 \\
\hline D6 & 130,617 & 5,440 & 0,091 & 224,370 \\
\hline D7 & 130,617 & 11,910 & 0,199 & 133,073 \\
\hline D8 & 130,617 & 3,950 & 0,066 & 277,736 \\
\hline D9 & 130,617 & 3,950 & 0,066 & 277,736 \\
\hline D10 & 130,617 & 11,910 & 0,199 & 133,073 \\
\hline D11 & 130,617 & 11,440 & 0,191 & 136,693 \\
\hline D12 & 130,617 & 11,440 & 0,191 & 136,693 \\
\hline D13 & 130,617 & 93,880 & 1,565 & 33,598 \\
\hline D14 & 130,617 & 31,920 & 0,532 & 68,969 \\
\hline D15 & 130,617 & 31,920 & 0,532 & 68,969 \\
\hline
\end{tabular}

Source: Calculation Analysis Results

\subsubsection{Distribution of Catchment Areas}

Catchment area calculations are done using the help of Autocad software. The catchment area can be seen in Figure 4.1, and the area of drainage catchment area can be seen in table 4.15.

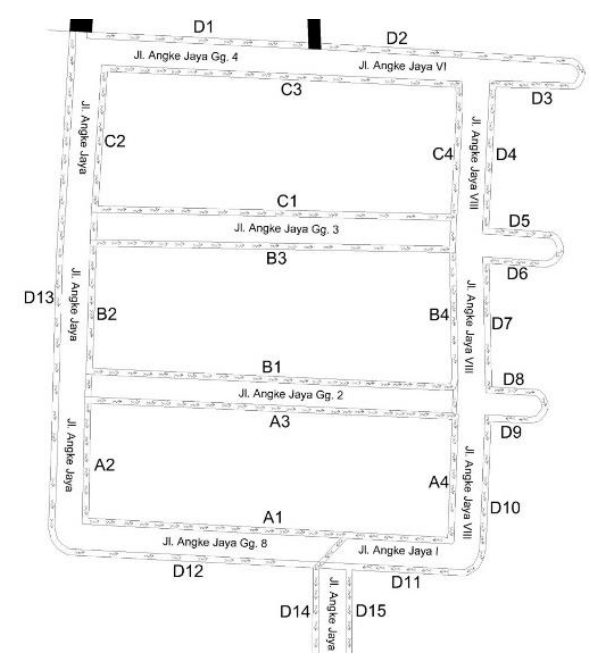

(Source: Personal Image)

Figure 3.1. Catchment Area 
Table 3.17. Capture Area of Drainage

\begin{tabular}{|c|c|c|c|c|c|c|}
\hline \multirow{2}{*}{$\begin{array}{c}\text { No. } \\
\text { Saluran }\end{array}$} & \multicolumn{2}{|c|}{ Jalan } & \multicolumn{2}{|c|}{ Pemukiman } & \multicolumn{2}{|c|}{ Jumlah } \\
\hline & $\mathbf{m}^{2}$ & $\mathbf{k m}^{2}$ & $\mathbf{m}^{2}$ & $\mathbf{k m}^{2}$ & $\mathbf{m}^{2}$ & $\mathbf{k m}^{2}$ \\
\hline A1 & 245,2375 & 0,00025 & 2268 & 0,0023 & 2513,238 & 0,002513 \\
\hline A 2 & 226,5083 & 0,00023 & 536 & 0,0005 & 762,5083 & 0,000763 \\
\hline $\mathrm{A} 3$ & 277,49 & 0,00028 & 2866 & 0,0029 & 3143,49 & 0,003143 \\
\hline $\mathrm{A} 4$ & 120,025 & 0,00012 & 705 & 0,0007 & 825,025 & 0,000825 \\
\hline B1 & 277,49 & 0,00028 & 1969 & 0,0020 & 2246,49 & 0,002246 \\
\hline B2 & 226,5083 & 0,00023 & 630 & 0,0006 & 856,5083 & 0,000857 \\
\hline B3 & 367,0625 & 0,00037 & 2091 & 0,0021 & 2458,063 & 0,002458 \\
\hline B4 & 120,025 & 0,00012 & 576 & 0,0006 & 696,025 & 0,000696 \\
\hline $\mathrm{C} 1$ & 367,0625 & 0,00037 & 1962 & 0,0020 & 2329,063 & 0,002329 \\
\hline $\mathrm{C} 2$ & 226,5083 & 0,00023 & 596 & 0,0006 & 822,5083 & 0,000823 \\
\hline $\mathrm{C} 3$ & 306,125 & 0,00031 & 1825 & 0,0018 & 2131,125 & 0,002131 \\
\hline $\mathrm{C} 4$ & 120,025 & 0,00012 & 519 & 0,0005 & 639,025 & 0,000639 \\
\hline D1 & 153,0625 & 0,00015 & 987 & 0,0010 & 1140,063 & 0,00114 \\
\hline $\mathrm{D} 2$ & 153,0625 & 0,00015 & 852 & 0,0009 & 1005,063 & 0,001005 \\
\hline D3 & 96,2325 & 0,00010 & 339 & 0,0003 & 435,2325 & 0,000435 \\
\hline D4 & 120,025 & 0,00012 & 537 & 0,0005 & 657,025 & 0,000657 \\
\hline D5 & 116,25 & 0,00012 & 379 & 0,0004 & 495,25 & 0,000495 \\
\hline D6 & 116,25 & 0,00012 & 314 & 0,0003 & 430,25 & 0,00043 \\
\hline D7 & 120,025 & 0,00012 & 441 & 0,0004 & 561,025 & 0,000561 \\
\hline D8 & 62,715 & 0,00006 & 154 & 0,0002 & 216,715 & 0,000217 \\
\hline D9 & 62,715 & 0,00006 & 277 & 0,0003 & 339,715 & 0,00034 \\
\hline D10 & 120,025 & 0,00012 & 405 & 0,0004 & 525,025 & 0,000525 \\
\hline D11 & 357,975 & 0,00036 & 353 & 0,0004 & 710,975 & 0,000711 \\
\hline D12 & 66,25 & 0,00007 & 618 & 0,0006 & 684,25 & 0,000684 \\
\hline D13 & 679,525 & 0,00068 & 3066 & 0,0031 & 3745,525 & 0,003746 \\
\hline D14 & 274,1875 & 0,00027 & 919 & 0,0009 & 1193,188 & 0,001193 \\
\hline D15 & 274,1875 & 0,00027 & 891 & 0,0009 & 1165,188 & 0,001165 \\
\hline
\end{tabular}

Source: Calculation Analysis Results

\subsubsection{Calculation of Rain Water Flow Discharge}

Tabel 3.18. Rainwater Flow Discharge

\begin{tabular}{|c|c|c|c|c|c|}
\hline \multirow{2}{*}{$\begin{array}{c}\text { No } \\
\text { Saluran }\end{array}$} & \multirow{2}{*}{ C } & I & $\mathrm{A}$ & Qp & Qp total \\
\hline & & $\mathrm{mm} / \mathrm{jam}$ & $\mathrm{km}^{2}$ & $\mathrm{~m} 3 / \mathrm{det}$ & $\mathrm{m} 3 / \mathrm{det}$ \\
\hline A1 & 0,75 & 86,011 & 0,00251 & 0,04507 & 0,06108 \\
\hline A2 & 0,75 & 100,673 & 0,00076 & 0,01602 & 0,09635 \\
\hline A3 & 0,75 & 55,692 & 0,00314 & 0,03650 & 0,07176 \\
\hline A4 & 0,75 & 29,141 & 0,00083 & 0,00501 & 0,19424 \\
\hline B1 & 0,75 & 55,692 & 0,00225 & 0,02608 & 0,06134 \\
\hline B2 & 0,75 & 100,673 & 0,00086 & 0,01799 & 0,03526 \\
\hline B3 & 0,75 & 54,477 & 0,00246 & 0,02792 & 0,04519 \\
\hline B4 & 0,75 & 132,998 & 0,0007 & 0,01930 & 0,09139 \\
\hline $\mathrm{C} 1$ & 0,75 & 54,477 & 0,00233 & 0,02645 & 0,04373 \\
\hline $\mathrm{C} 2$ & 0,75 & 100,673 & 0,00082 & 0,01728 & 0,01728 \\
\hline C3 & 0,75 & 55,998 & 0,00213 & 0,02488 & 0,02488 \\
\hline $\mathrm{C} 4$ & 0,75 & 132,998 & 0,00064 & 0,01772 & 0,04260 \\
\hline D1 & 0,75 & 22,87 & 0,00114 & 0,00544 & 0,00544 \\
\hline D2 & 0,75 & 22,829 & 0,00101 & 0,00478 & \begin{tabular}{|l}
0,01022 \\
\end{tabular} \\
\hline D3 & 0,75 & 223,277 & 0,00044 & 0,02025 & 0,03047 \\
\hline D4 & 0,75 & 133,073 & 0,00066 & 0,01823 & 0,04870 \\
\hline D5 & 0,75 & 224,37 & 0,0005 & 0,02316 & 0,07186 \\
\hline D6 & 0,75 & 224,37 & 0,00043 & 0,02012 & 0,09197 \\
\hline D7 & 0,75 & 133,073 & 0,00056 & 0,01557 & 0,10754 \\
\hline D8 & 0,75 & 277,736 & 0,00022 & 0,01257 & 0,12010 \\
\hline D9 & 0,75 & 277,736 & 0,00034 & 0,01969 & 0,13979 \\
\hline D10 & 0,75 & 133,073 & 0,00053 & 0,01457 & 0,15436 \\
\hline D11 & 0,75 & 136,693 & 0,00071 & 0,02026 & 0,17462 \\
\hline D12 & 0,75 & 136,693 & 0,00068 & 0,01949 & 0,04574 \\
\hline D13 & 0,75 & 33,598 & 0,00375 & 0,02624 & 0,02624 \\
\hline D14 & 0,75 & 68,969 & 0,00119 & 0,01716 & 0,06289 \\
\hline D15 & 0,75 & 68,969 & 0,00117 & 0,01675 & 0,19137 \\
\hline
\end{tabular}

Source: Calculation Analysis Results

Calculation of rainwater flowrate using the rational method formula, the following is the calculation of rainwater flowrate for channel $\mathrm{D} 2$, which is as follows:

$\mathrm{Qp}=0.278$ C.I.A 
$\mathrm{Qp}=0.278 .(0.75) .(22.829) .(0.00101)$

$\mathrm{Qp}=0.01022 \mathrm{~m} 3 / \mathrm{sec}$

For channel D2, an additional water discharge from D1 flow is added,

Qp D1 + Q_p D2 $=0.00544+0.00478$

Qtotal D2 $=0.01022 \mathrm{~m} 3 / \mathrm{sec}$

The full calculation will be presented in table 4.18 .

\subsubsection{Calculation of Dirty Water Discharge}

The calculated dirty water discharge is the water debit that comes from household waste, and other buildings. The amount is affected by the large number of residents and the average water needs of the population. Estimates for the average disposal of liquid waste per person per day are presented in table 2.10, and it is concluded that the amount of waste water per person per day is 400 liters. Following are the calculations for channel D2.

Q_ak = Pn x 400 liters / person / day

Q_ak = Pn x 0.00463 liter / person / sec

Q_ak $=45 \times 0.00463$ liter / person / sec

Q_ak $=0.2083 \mathrm{~m} 3 / \mathrm{sec}$

The complete calculation for dirty water discharge has been presented in table 3.19.

Tabel 3.19. Debit Air Kotor

\begin{tabular}{|c|c|c|c|c|c|c|}
\hline \multirow{2}{*}{$\begin{array}{l}\text { No. } \\
\text { Saluran }\end{array}$} & \multirow{2}{*}{$\begin{array}{l}\text { Jumlah } \\
\text { Rumah }\end{array}$} & \multirow{2}{*}{$\begin{array}{c}\text { Jumlah } \\
\text { Orang }\end{array}$} & $\begin{array}{l}\text { Air } \\
\text { Buangan }\end{array}$ & $\begin{array}{l}\text { Air } \\
\text { Buangan }\end{array}$ & Qak & $\begin{array}{l}\text { Qak } \\
\text { Total }\end{array}$ \\
\hline & & & 1t/org/hari & 1t/org/dtk & $\mathrm{m}^{3 /} / \mathrm{dt}$ & $\mathrm{m}^{3 /} / \mathrm{dt}$ \\
\hline A1 & 17 & 68 & 400 & 0,00463 & 0,3148 & 0,356 \\
\hline $\mathrm{A} 2$ & 2 & 9 & 400 & 0,00463 & 0,0417 & 0,435 \\
\hline $\mathrm{A} 3$ & 17 & 66 & 400 & 0,00463 & 0,3056 & 0,384 \\
\hline $\mathrm{A} 4$ & 2 & 10 & 400 & 0,00463 & 0,0463 & 1,398 \\
\hline B1 & 18 & 70 & 400 & 0,00463 & 0,3241 & 0,403 \\
\hline B2 & 2 & 9 & 400 & 0,00463 & 0,0417 & 0,079 \\
\hline B3 & 18 & 61 & 400 & 0,00463 & 0,2824 & 0,319 \\
\hline B4 & 2 & 10 & 400 & 0,00463 & 0,0463 & 0,644 \\
\hline $\mathrm{C} 1$ & 18 & 59 & 400 & 0,00463 & 0,2731 & 0,310 \\
\hline $\mathrm{C} 2$ & 2 & 8 & 400 & 0,00463 & 0,0370 & 0,037 \\
\hline $\mathrm{C} 3$ & 17 & 58 & 400 & 0,00463 & 0,2685 & 0,269 \\
\hline $\mathrm{C} 4$ & 2 & 9 & 400 & 0,00463 & 0,0417 & 0,310 \\
\hline D1 & 10 & 48 & 400 & 0,00463 & 0,2222 & 0,222 \\
\hline D2 & 9 & 45 & 400 & 0,00463 & 0,2083 & 0,431 \\
\hline D3 & 2 & 8 & 400 & 0,00463 & 0,0370 & 0,468 \\
\hline D4 & 2 & 7 & 400 & 0,00463 & 0,0324 & 0,500 \\
\hline D5 & 3 & 9 & 400 & 0,00463 & 0,0417 & 0,542 \\
\hline D6 & 3 & 8 & 400 & 0,00463 & 0,0370 & 0,579 \\
\hline D7 & 3 & 7 & 400 & 0,00463 & 0,0324 & 0,611 \\
\hline D8 & 3 & 9 & 400 & 0,00463 & 0,0417 & 0,653 \\
\hline D9 & 3 & 9 & 400 & 0,00463 & 0,0417 & 0,694 \\
\hline D10 & 5 & 20 & 400 & 0,00463 & 0,0926 & 0,787 \\
\hline D11 & 4 & 18 & 400 & 0,00463 & 0,0833 & 0,870 \\
\hline D12 & 12 & 48 & 400 & 0,00463 & 0,2222 & 0,481 \\
\hline D13 & 18 & 56 & 400 & 0,00463 & 0,2593 & 0,259 \\
\hline D14 & 13 & 52 & 400 & 0,00463 & 0,2407 & 0,722 \\
\hline D15 & 11 & 44 & 400 & 0,00463 & 0,2037 & 1,074 \\
\hline
\end{tabular}

Source: Calculation Analysis Results

\subsubsection{Calculation of Flood Discharge Plan}

In the calculation of flood discharges in the Angke Jaya Tambora Housing Area of West Jakarta, namely rainwater flow discharges added with dirty water discharge.

The following is a calculation on channel A1.

$\mathrm{Q}_{\mathrm{r}}=\mathrm{Q}_{\mathrm{P} \text { total }}+\mathrm{Q}_{\mathrm{ak} \text { total }}$

$\mathrm{Q}_{\mathrm{r}}=0,00053+0.042$

$\mathrm{Q}_{\mathrm{r}}=0.043 \mathrm{~m} 3 / \mathrm{dt}$

Complete calculations for flood discharge are presented in table 3.20. 
Table 3.20. Flood Discharge Plan

\begin{tabular}{|c|c|c|c|}
\hline \multirow{2}{*}{$\begin{array}{c}\text { No } \\
\text { Saluran }\end{array}$} & Qp total & $\begin{array}{c}\text { Qak } \\
\text { total }\end{array}$ & Qr \\
\cline { 2 - 4 } & $\mathrm{m}^{3} / \mathrm{dt}$ & $\mathrm{m}^{3} / \mathrm{dt}$ & $\mathrm{m}^{3} / \mathrm{dt}$ \\
\hline A1 & 0,06108 & 0,356 & 0,418 \\
\hline A2 & 0,09635 & 0,435 & 0,532 \\
\hline A3 & 0,07176 & 0,384 & 0,456 \\
\hline A4 & 0,19424 & 1,398 & 1,592 \\
\hline B1 & 0,06134 & 0,403 & 0,464 \\
\hline B2 & 0,03526 & 0,079 & 0,114 \\
\hline B3 & 0,04519 & 0,319 & 0,365 \\
\hline B4 & 0,09139 & 0,644 & 0,735 \\
\hline C1 & 0,04373 & 0,310 & 0,354 \\
\hline C2 & 0,01728 & 0,037 & 0,054 \\
\hline C3 & 0,02488 & 0,269 & 0,293 \\
\hline C4 & 0,04260 & 0,310 & 0,353 \\
\hline D1 & 0,00544 & 0,222 & 0,228 \\
\hline D2 & 0,01022 & 0,431 & 0,441 \\
\hline D3 & 0,03047 & 0,468 & 0,498 \\
\hline D4 & 0,04870 & 0,500 & 0,549 \\
\hline D5 & 0,07186 & 0,542 & 0,614 \\
\hline D6 & 0,09197 & 0,579 & 0,671 \\
\hline D7 & 0,10754 & 0,611 & 0,719 \\
\hline D8 & 0,12010 & 0,653 & 0,773 \\
\hline D9 & 0,13979 & 0,694 & 0,834 \\
\hline D10 & 0,15436 & 0,787 & 0,941 \\
\hline D11 & 0,17462 & 0,870 & 1,045 \\
\hline D12 & 0,04574 & 0,481 & 0,527 \\
\hline D13 & 0,02624 & 0,259 & 0,286 \\
\hline D14 & 0,06289 & 0,722 & 0,785 \\
\hline D15 & 0,19137 & 1,074 & 1,265 \\
\hline
\end{tabular}

Source: Calculation Analysis Results

\subsection{Calculation of Drainage Channel Dimensions}

After knowing the planned flood discharge in the West Jakarta Angora Jaya Tambora Housing Area, the dimensions of the existing drainage channel will be calculated to determine whether or not the channel is sufficient to accommodate the planned flood discharge. Furthermore, if there are unsafe channels, a new drainage channel calculation will be performed to determine the dimensions of the safe channel.

\subsubsection{Calculation of Existing Drainage Channel Dimensions.}

$$
\mathrm{b}=0,6 \text { Berikut merupakan perhitungan untuk saluran A1 : }
$$

$\mathrm{h}=1$

$\mathrm{S}=0,02$

$\mathrm{A}=\mathrm{b} \times \mathrm{h}$

$\mathrm{A}=0,6 \times 1=0,6 \mathrm{~m} 2$

$\mathrm{P}=\mathrm{b}+2 \mathrm{~h}$

$P=0,6+(2 \times 1)=2,60$

$\mathrm{R}=\frac{\mathrm{A}}{\mathrm{P}}$

$\mathrm{R}=\frac{0,60}{2,60}=0,23 \mathrm{~m}$

$\mathrm{V}=\frac{1}{\mathrm{n}} \mathrm{R}^{\frac{2}{3}} \mathrm{~S}^{\frac{1}{2}}$

$\mathrm{V}=\frac{1}{0,01} 0,23^{\frac{2}{3}} \cdot 0,02^{\frac{1}{2}}$

$\mathrm{V}=3,75 \mathrm{~m} / \mathrm{sec}$ 
$\mathrm{Q}_{\mathrm{sal}}=\mathrm{AxV}=0,6 \times 3,75=2,25 \mathrm{~m}^{3} / \mathrm{dt}$

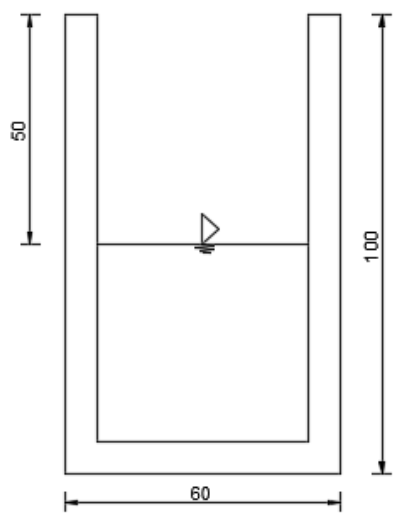

(Source: Personal Image)

Figure 3.2. Drainage Channel Cut

If Qsaluran > Qrencana, the drainage channel is able to accommodate the flow of flood discharge, and declared safe. If the Channel <Qrage drainage plan is able to accommodate the flow of flood discharge, and declare dunsafe. The full calculation is presented in table 3.21.

Table 3.21. Calculation of Existing Drainage Channel Dimensions

\begin{tabular}{|c|c|c|c|c|c|c|c|c|}
\hline & & & & & & & & \\
\hline $\begin{array}{c}\text { No } \\
\text { Saluran }\end{array}$ & \begin{tabular}{|c|}
$\mathrm{b}$ \\
(m) \\
\end{tabular} & \begin{tabular}{|c|}
$\mathrm{h}$ \\
$(\mathrm{m})$ \\
\end{tabular} & \begin{tabular}{|l|}
$\mathrm{A}$ \\
$\left(\mathrm{m}^{2}\right)$ \\
\end{tabular} & \begin{tabular}{|c|}
$\mathrm{V}$ \\
$(\mathrm{m} / \mathrm{d})$ \\
\end{tabular} & 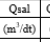 & \begin{tabular}{|l|} 
Qrencena \\
$\left(\mathrm{m}^{1} / \mathrm{det}\right)$ \\
\end{tabular} & Keterangan & Keteran \\
\hline A1 & 0,60 & 1,00 & 0,60 & 3,75 & 2,25 & 0,4 & $\begin{array}{l}\text { Qsess } \\
\text { ren }\end{array}$ & Amax \\
\hline A2 & 0,70 & 0,35 & 0,25 & 2,68 & 0,66 & 0,53 & Qrenc & Amax \\
\hline A3 & \begin{tabular}{|l|}
0,80 \\
\end{tabular} & 0,50 & \begin{tabular}{|l|}
0,40 \\
\end{tabular} & 2,46 & 0,98 & 0,46 & & Amm \\
\hline A4 & \begin{tabular}{|l|}
0,80 \\
\end{tabular} & 0,50 & 0,40 & 1,69 & 0,68 & 1,59 & $\begin{array}{c}\text { Qsen } \\
\text { Qren }\end{array}$ & $\begin{array}{l}\text { Tididk } \\
\text { Amanan }\end{array}$ \\
\hline B1 & 0,80 & 0,50 & \begin{tabular}{|l|l|} 
\\
\end{tabular} & 2,46 & 0,98 & 0,46 & 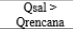 & Ams \\
\hline B2 & 0,70 & 0,35 & 0,25 & 2,68 & 0,66 & 0,11 & $\begin{array}{l}\text { QSas I } \\
\text { Qrencer }\end{array}$ & Ams \\
\hline B3 & 0,80 & 0,60 & \begin{tabular}{|l|l|}
0,48 \\
\end{tabular} & 2,43 & 1,17 & 0,36 & $\begin{array}{l}\text { Qsal } \\
\text { Orencan }\end{array}$ & $\operatorname{Aman}$ \\
\hline B4 & \begin{tabular}{|l|l|}
0,80 \\
\end{tabular} & 0,60 & \begin{tabular}{|l|}
0,48 \\
\end{tabular} & 3,61 & 1,73 & 0,73 & $\begin{array}{c}\text { Qsen } \\
\text { Qren }\end{array}$ & $A_{m z}$ \\
\hline $\mathrm{C} 1$ & 0,80 & 0,60 & \begin{tabular}{|l|} 
\\
\end{tabular} & 2,43 & 1,17 & 0,3 & $\begin{array}{l}\text { Qsal } \\
\text { Qreaceas }\end{array}$ & Ams \\
\hline $\mathrm{C}_{2}$ & 0,50 & 0,60 & \begin{tabular}{|l|}
, 30 \\
\end{tabular} & 2,70 & 0,81 & 0,05 & $\begin{array}{l}\text { Qsal > } \\
\text { Qrencana }\end{array}$ & Aman \\
\hline C3 & 0,80 & 0,60 & \begin{tabular}{|l|}
0,48 \\
\end{tabular} & 2,47 & 1,18 & 0,2 & $\begin{array}{l}\text { Qsal } \\
\text { Qrencana }\end{array}$ & $\mathrm{A}_{\mathrm{m} 2}$ \\
\hline C4 & \begin{tabular}{|l|l|}
0,80 \\
\end{tabular} & 0,60 & \begin{tabular}{|l|}
0,48 \\
\end{tabular} & 3,61 & 1,73 & 0,35 & $\begin{array}{l}\text { Qsal } \\
\text { Qrencan }\end{array}$ & Ama \\
\hline D1 & 0,70 & 0,80 & \begin{tabular}{|l|}
0,56 \\
\end{tabular} & 1,59 & 0,89 & 0,23 & $\begin{array}{l}\text { Qsal } \\
\text { Qrencana }\end{array}$ & $A_{\operatorname{man}}$ \\
\hline D2 & 0,60 & 0,60 & \begin{tabular}{|l|}
0,36 \\
\end{tabular} & 1,40 & 0,50 & 0,4 & QR & Ams \\
\hline D3 & \begin{tabular}{|l|}
0,40 \\
\end{tabular} & 0,50 & 0,20 & 3,49 & 0,70 & 0,50 & & Ams \\
\hline D4 & \begin{tabular}{|l|}
0,80 \\
\end{tabular} & 0,60 & \begin{tabular}{|l|}
0,48 \\
\end{tabular} & 3,80 & 1,83 & 0,55 & & Amax \\
\hline D5 & \begin{tabular}{|l|}
0,40 \\
\end{tabular} & 0,40 & \begin{tabular}{|l|}
0,16 \\
\end{tabular} & 3,35 & 0,54 & 0,6 & $\begin{array}{l}\text { Qseal < } \\
\text { Orencana }\end{array}$ & $\begin{array}{l}\text { Tidak } \\
\text { Amam }\end{array}$ \\
\hline D6 & \begin{tabular}{|l|l|}
0,40 \\
\end{tabular} & 0,40 & \begin{tabular}{|l|}
0,16 \\
\end{tabular} & 3,35 & 0,54 & 0,67 & & $\begin{array}{l}\text { Tidial } \\
\text { Amman }\end{array}$ \\
\hline D? & 0,80 & 0,60 & \begin{tabular}{|l|}
0,48 \\
\end{tabular} & 3,80 & 1,83 & 0,72 & & $A \operatorname{man}$ \\
\hline D8 & $\begin{array}{l}, 30 \\
\end{array}$ & 0,40 & \begin{tabular}{|l|}
0,12 \\
\end{tabular} & 3,26 & 0,39 & 0,77 & $\begin{array}{l}\text { Qseal < } \\
\text { Orencan }\end{array}$ & $\begin{array}{l}\text { Tidakk } \\
\text { Amam }\end{array}$ \\
\hline D9 & $|0,30|$ & $|0,40|$ & $|0,12|$ & $|3,26|$ & 0,39 & 0,83 & $\begin{array}{c}\text { Qsal < } \\
\text { Orencena }\end{array}$ & $\begin{array}{l}\text { ridiak } \\
\text { max }\end{array}$ \\
\hline D10 & 0,80 & 0,60 & \begin{tabular}{|l|l|}
0,48 \\
\end{tabular} & 3,80 & 1,83 & 0,94 & & Aman \\
\hline D11 & $0,80 \mid$ & 0,70 & \begin{tabular}{|l|}
0,56 \\
\end{tabular} & 4,01 & 2,24 & 1,04 & & Ama \\
\hline D12 & \begin{tabular}{|l|}
0,80 \\
\end{tabular} & 0,70 & \begin{tabular}{|l|}
0,56 \\
\end{tabular} & 4,01 & 2,24 & 0,53 & & Amax \\
\hline D13 & \begin{tabular}{|l|}
0,60 \\
\end{tabular} & 0,60 & \begin{tabular}{|l|}
0,36 \\
\end{tabular} & 1,69 & 0,61 & 0,29 & & Aman \\
\hline D14 & \begin{tabular}{|l|}
0,70 \\
\end{tabular} & 0,80 & \begin{tabular}{|l|}
0,56 \\
\end{tabular} & 2,76 & 1,55 & 0,79 & & $\mathrm{~A}_{\max }$ \\
\hline D15 & 0,70 & 0,80 & \begin{tabular}{|l|}
0,56 \\
\end{tabular} & 2,76 & 1,55 & 1,27 & rencan & $A_{m z}$ \\
\hline
\end{tabular}

Source: Calculation Analysis Results

Berdasarkan perhitungan dimensi saluran drainase eksisting pada tabel 3.21, ditemukan saluran yang masuk dalam kategori "tidak aman", karena kapasitas saluran tidak cukup untuk menampung debit banjir rencana, yaitu pada saluran A4, D5, D6, D8, dan D9. Dalam mengatasi hal ini akan direncanakan sumur resapan.

\subsection{Infiltration Wells Plan}

The infiltration well planning is used in draining excess water discharge in several "unsafe" channels, by draining rainwater that falls from the roofs of residents' homes to seep into the ground by storing the water in the infiltration well. Channels that fall into the "unsafe" category will be presented in table 3.22 , as follows : 
Table 3.22 Channels By Unsafe Categories (Q drainage channels <Q plan)

\begin{tabular}{|c|c|c|c|c|}
\hline \multirow{2}{*}{$\begin{array}{c}\text { No } \\
\text { Saluran }\end{array}$} & Qsal & Qrencana & $\begin{array}{c}\text { Kelebihan } \\
\text { Debit }\end{array}$ & Kategori \\
\cline { 2 - 4 } & $\mathrm{m}^{3} / \mathrm{dt}$ & $\mathrm{m}^{3} / \mathrm{det}$ & $\mathrm{m}^{3} /$ det & \\
\hline A4 & 0,68 & 1,59 & 0,92 & $\begin{array}{c}\text { Tidak } \\
\text { Aman }\end{array}$ \\
\hline D5 & 0,54 & 0,61 & 0,08 & $\begin{array}{c}\text { Tidak } \\
\text { Aman }\end{array}$ \\
\hline D6 & 0,54 & 0,67 & 0,13 & $\begin{array}{c}\text { Tidak } \\
\text { Aman }\end{array}$ \\
\hline D8 & 0,39 & 0,77 & 0,38 & $\begin{array}{c}\text { Tidak } \\
\text { Aman }\end{array}$ \\
\hline D9 & 0,39 & 0,83 & 0,44 & $\begin{array}{c}\text { Tidak } \\
\text { Aman }\end{array}$ \\
\hline
\end{tabular}

Source: Calculation Analysis Results

Based on table 3.22, there are channels that fall into the "unsafe" category, so that an infiltration well will be planned.

\subsubsection{Infiltration Wells Construction}

1. Channels of income / expenditure using pralon pipes.

2. The well wall can use masonry without plastering

3. The bottom of the well and the gaps between the soil excavation and the wall where the water is absorbed are

filled with fibers / gravel.

\subsubsection{Discharge of Rainfall Plans on the Roof Surface}

To find out the flow of rain that enters the infiltration well, it is necessary to know in advance the flow of rain falling through the roof of the residents' houses. Example calculation using No. A4 channels as follows:

$\mathrm{Q}$ masuk $=0.278$. C.I.A

Is known :

Catap $=0.75$ (based on Table 2.9. Runoff Coefficient Standard)

IA $4=29.14 \mathrm{~mm} /$ hour

Aatap $=80 \mathrm{~m} 2=0.0080 \mathrm{~km} 2$

So, Q masuk is:

Qmasuk $=0.278 \cdot 0.75 \cdot 29.14 \cdot 0.008$

$=0.048607 \mathrm{~m} 3 / \mathrm{sec}$

Calculation of rain discharges that fall to the roof surface will be presented in full in table 3.23 , as follows :

Table 3.23 Rainwater Discharge Falls on the Roof

\begin{tabular}{|c|c|c|c|c|c|}
\hline $\begin{array}{c}\text { No } \\
\text { Saluran }\end{array}$ & C & I & Aatap & Aatap & Qmasuk \\
\cline { 3 - 6 } & & $\mathrm{mm} / \mathrm{jam}$ & $\mathrm{m}^{2}$ & $\mathrm{~km}^{2}$ & $\mathrm{~m}^{3} /$ det \\
\hline A4 & 0,75 & 29,14 & 80,00 & 0,008 & 0,048607 \\
\hline D5 & 0,75 & 224,37 & 80,00 & 0,008 & 0,374249 \\
\hline D6 & 0,75 & 224,37 & 80,00 & 0,008 & 0,374249 \\
\hline D8 & 0,75 & 277,736 & 80,00 & 0,008 & 0,463264 \\
\hline D9 & 0,75 & 277,736 & 80,00 & 0,008 & 0,463264 \\
\hline
\end{tabular}

Source: Calculation Analysis Results

\subsubsection{Discharge of Absorption Well Absorption}

Furthermore, it is necessary to know the discharge of rainwater that enters the absorption well (Qresapan).

The calculation refers to No. Channel A4, as follows : 


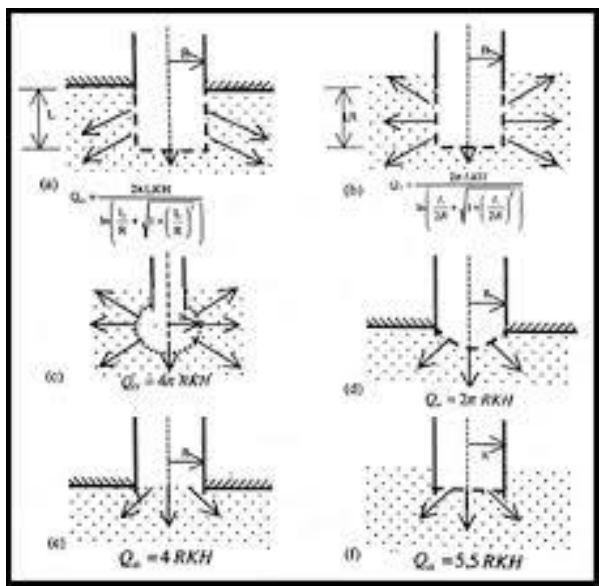

(Source: Sistem Drainase Perkotaan yang Berkelanjutan, 2004:300)

Figure 3.3 Geometric Factors of Infiltration Wells

Qresapan $=$ F. K. H

Infiltration Wells Design Plan:

Is known :

- Qresapan = Discharge that can be absorbed by infiltration wells (m3 / sec)

- Type of blank wells with circular appearance

- The planned well diameter is $2 \mathrm{~m}=\mathrm{R}=1 \mathrm{~m}$

- $\mathrm{F}=$ geometric $/$ circumference factor $==2 \times 3.14 \times 1=3.14 \mathrm{~m}$

- $\mathrm{K}=$ For the soil permeability value, it is assumed that the value of $\mathrm{K}=10-2 \mathrm{~cm} / \mathrm{s}=10-4 \mathrm{~m} / \mathrm{s}$ for the shaft soil.

\begin{tabular}{|l|l|l|}
\hline \multicolumn{1}{|c|}{ JENIS TANAH } & \multicolumn{1}{|c|}{$\mathbf{k}(\mathbf{c m} /$ det $)$} & \multicolumn{1}{c|}{ NAMA } \\
\hline Kerikil & $>10^{-1}$ & High permeability \\
\hline kerikil halus/pasir & $10^{-1}-10^{-3}$ & Mediumpermeability \\
\hline $\begin{array}{l}\text { pasir sangat halus } \\
\text { pasir lanau } \\
\text { lanau tidak padat }\end{array}$ & $10^{-3}-10^{-5}$ & Low permeability \\
\hline $\begin{array}{l}\text { lanaupadat } \\
\text { lanau lempung } \\
\text { lanau tidak murni }\end{array}$ & $10^{-5}-10^{-7}$ & Verylow permeability \\
\hline Lempung & $<10^{-7}$ & Impervious (rapatair) \\
\hline
\end{tabular}

(Source: www.google.co.id)

Figure 3.4 Soil Permeability Coefficient

- $\mathrm{H}=$ Assumption of infiltration well depth $=2 \mathrm{~m}$

Infiltration Wells Calculation:

Qresapan = F. K. H

$=3.14 .10-4.2$

$=0,000628 \mathrm{~m} 3 / \mathrm{sec}$

For complete Qresapan calculation will be presented in table 4.24, as follows : 
Table 3.24 Qresapan Rainwater in Infiltration Wells

\begin{tabular}{|c|c|c|c|c|}
\hline \multirow{2}{*}{$\begin{array}{c}\text { No } \\
\text { Saluran }\end{array}$} & \multicolumn{4}{|c|}{ Qresapan Air Hujan dalam Sumur } \\
\cline { 2 - 5 } & $\begin{array}{c}\mathrm{F} \\
(\mathrm{m})\end{array}$ & $\begin{array}{c}\mathrm{K} \\
(\mathrm{cm} / \mathrm{dtk})\end{array}$ & $\begin{array}{c}\mathrm{H} \\
(\mathrm{m})\end{array}$ & $\begin{array}{c}\text { Qresapan } \\
\left(\mathrm{m}^{3} / \mathrm{dtk}\right)\end{array}$ \\
\hline A4 & 3,14 & 0,0001 & 2,00 & 0,000628 \\
\hline D5 & 3,14 & 0,0001 & 2,00 & 0,000628 \\
\hline D6 & 3,14 & 0,0001 & 2,00 & 0,000628 \\
\hline D8 & 3,14 & 0,0001 & 2,00 & 0,000628 \\
\hline D9 & 3,14 & 0,0001 & 2,00 & 0,000628 \\
\hline
\end{tabular}

Source : Calculation Analysis Results

\subsubsection{Residual Water Discharge Flowing into Drainage Channels}

Furthermore, it will be found the difference in the remaining discharge from the rain discharge that has been accommodated into the infiltration well, by way of Qmasuk (Table 4.23) reduced by Qresapan (Table 4.24). The following is an example of a calculation that refers to No. A4 channel:

Is known :

$\mathrm{Q}$ in $=0.048607 \mathrm{~m} 3 / \mathrm{sec}($ table 4.23$)$

$\mathrm{Q}$ infiltration $=0,000628$ (table 4.24)

Settlement:

Qtampung = Qmasuk - Qresapan

$$
\begin{aligned}
& =0.048607-0.000628 \\
& =0.047979 \mathrm{~m} 3 / \mathrm{sec}
\end{aligned}
$$

For the calculation of the complete infiltration wells collected discharge will be presented in table 4.25 as follows :

Table 3.25 Discharge accommodated in infiltration wells

\begin{tabular}{|c|c|c|c|}
\hline $\begin{array}{c}\text { No } \\
\text { Saluran }\end{array}$ & $\begin{array}{c}\text { Qmasuk } \\
\text { atap } \\
\left(\mathrm{m}^{3} / \mathrm{dtk}\right) \\
(4)\end{array}$ & $\begin{array}{c}\text { Qresapan } \\
\left(\mathrm{m}^{3} / \mathrm{dtk}\right) \\
(5)\end{array}$ & $\begin{array}{c}\text { Qtampung } \\
\left(\mathrm{m}^{3} / \mathrm{dtk}\right) \\
(6)=(5)- \\
(4)\end{array}$ \\
\hline A4 & 0,048607 & 0,000628 & 0,047979 \\
\hline D5 & 0,374249 & 0,000628 & 0,373621 \\
\hline D6 & 0,374249 & 0,000628 & 0,373621 \\
\hline D8 & 0,463264 & 0,000628 & 0,462636 \\
\hline D9 & 0,463264 & 0,000628 & 0,462636 \\
\hline
\end{tabular}

Source : Calculation Analysis Results

\subsubsection{Total Infiltration Wells Needs}

After knowing the difference in the remaining discharge from the rain discharge that has been accommodated into the infiltration well, it can be seen the amount of infiltration well needs in the area of the house in each Channel Number. Example calculation refers to No. A4 channel:

Is known :

Discharge Excess A4 $=0.92 \mathrm{~m} 3 / \mathrm{sec}$ (Table 4.22)

Qtampung $=0.047979 \mathrm{~m} 3 / \mathrm{sec}$

Settlement :

Jumlah Sumur Resapan $=\frac{\text { Kelebihan Debit }}{\text { Qtampung }}$

The calculation of the number of recharge well requirements in full is presented in table 4.26 as follows : 


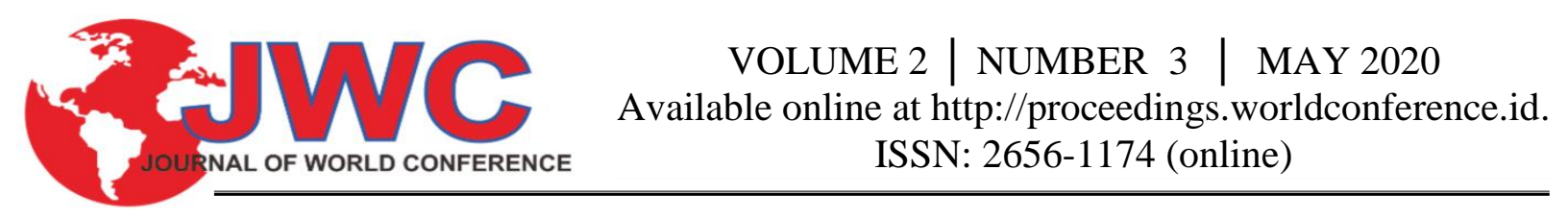

Table 3.26 Amount of Infiltration Wells Needs

\begin{tabular}{|c|c|c|c|}
\hline $\begin{array}{c}\text { No } \\
\text { Saluran }\end{array}$ & $\begin{array}{c}\text { Qlebih } \\
\left(\mathrm{m}^{3} / \mathrm{dtk}\right) \\
(3)= \\
(2)-(1)\end{array}$ & $\begin{array}{c}\text { Qtampung } \\
\left(\mathrm{m}^{3} / \mathrm{dtk}\right) \\
(6)=(5)- \\
(4)\end{array}$ & $\begin{array}{c}\text { Jumlah } \\
\text { Sumur } \\
\text { Resapan } \\
(7)= \\
(2):(6)\end{array}$ \\
\hline A4 & 0,92 & 0,047979 & 19 \\
\hline D5 & 0,08 & 0,373621 & 1 \\
\hline D6 & 0,13 & 0,373621 & 1 \\
\hline D8 & 0,38 & 0,462636 & 1 \\
\hline D9 & 0,44 & 0,462636 & 1 \\
\hline
\end{tabular}

Source : Calculation Analysis Results

From table 4.26, it is known that the number of infiltration wells needed in A4 Channel is 19 units, Channel D5, D6, D8, and D9 are 1 piece.

\section{Conclussion}

From the results of the analysis and discussion in the previous chapter and answer from the formulation of the problem, the following conclusions can be drawn:

1. The condition of the drainage canal in Angke Jaya Tambora Housing Area West Jakarta is poorly maintained, there are many houses that progress to cover the existing channels, making the channel difficult to clean, so there is a lot of garbage in the channel and accumulation of sedimentation. There are 5 channels that are concluded to be "unsafe", because the planned flood discharge is greater than the drainage capacity, namely on channels A4, D5, D6, D8, andD9.

2. The need for flood drainage capacity in the Angke Jaya Tambora Housing Area of West Jakarta is $13,225 \mathrm{~m} 3$ / second.5.2 Suggestions

Suggestions that can be delivered at the writing of this thesis after getting the results and solutions provided, the advice that will be given are as follows:

1. Regular cleaning of drainage channels, on sedimentation and rubbish in the drainage channel.

2. Construction of infiltration wells on channels A4, D5, D6, D8, D9 can be carried out, so that in the rainy season stagnant water can be diverted to infiltration wells.

\section{References}

Analisa Kapasitas Saluran Drainase Perumahan Kopri Kelurahan Kedaung Wetan Tanggerang. Wijaya, Sendi Eka. 2015. Jakarta : Universitas Mercubuana, 2015.

Analisis Hidrologi dan Kapasitas Drainase Kota Surakarta. Hutomo, Fajar Priyo and Firmansyah, Rheza. 2016. Semarang : Politeknik Negeri Semarang, 2016.

Pengelolaan Kualitas Air dan Pengendalian Pencemaran Air. Indonesia, Pemerintah Republik. 1982. Indonesia : Pemerintah Republik Indonesia, 1982. 82.

Perencanaan Saluran Drainase Ramah Lingkungan Dusun Kedungbendo Desa Gemekan Kecamatan Sooko Kabupaten Mojokerto. Kurniawan, Hendry. 2017. Semarang : Politeknik Negeri Semarang, 2017.

Rancangan Sumur Resapan Air Hujan Sebagai Salah Satu Usaha Konservasi Air Tanah di Perumahan Dayu Baru Kabupaten Sleman Daerah Istimewa Yogyakarta. Indramaya, Eka Ayu and L. Setyawan, Purnama Ig. 2013. Yogyakarta : Universitas Gajah Mada, 2013.

Suripin. 2004. Sustainable Urban Drainage System. Yogyakarta : C.V. Andi Offset, 2004.

Tata Cara Perencanaan Sumur Resapan Air Hujan Untuk Lahan Pekarangan. Wilayah, Departemen Permukiman dan Prasarana. 2000. 2000. 